\title{
Erratum to: Mineralogical and geochemical aspects of the petrogenesis of Pan African Dokhan Volcanics at Esh El Mellaha Area-NE Desert, Egypt
}

\author{
S. Alaabed • Mohamed EI Tokhi
}

Published online: 17 May 2014

(C) Saudi Society for Geosciences 2014

Erratum to: Arab J Geosci

DOI 10.1007/s12517-013-1170-4

Regrettably, the online version of this article contained an error. Table 5 was incorrectly captured. The correct Table 5 is given below.

The online version of the original article can be found at http://dx.doi.org/ 10.1007/s12517-013-1170-4.

S. Alaabed $(\bowtie) \cdot$ M. El Tokhi

Department of Geology, College of Science, United Arab Emirates

University, P.O. Box 15551, Al-Ain, United Arab Emirates

e-mail: s.alaabed@uaeu.ac.aess 
Table 5 Results of crystal fractionation least-squares modeling of Dokhan volcanics of the Esh El Mellaha, Eastern Desert, Egypt

\begin{tabular}{|c|c|c|c|c|c|c|}
\hline \multirow[t]{2}{*}{ Major oxides } & \multicolumn{3}{|c|}{ Fractional Phases } & \multirow[t]{2}{*}{ Observed Daughter } & \multirow[t]{2}{*}{ Observed Parent } & \multirow[t]{2}{*}{ Calculated Paren } \\
\hline & Amphiboles & Plagioclase & Magnetite & & & \\
\hline \multicolumn{7}{|c|}{ Stage no. 1 The composition of basalts was assumed to be the parental liquid; the composition of andesites was assumed to be the derivative } \\
\hline & & & & D1-An & P1-B & CP1 \\
\hline $\mathrm{SiO}_{2}$ & 42.32 & 49.72 & 0.67 & 59.98 & 49.97 & 50.13 \\
\hline $\mathrm{TiO}_{2}$ & 1.39 & - & 11.4 & 1.27 & 2.01 & 1.96 \\
\hline $\mathrm{Al}_{2} \mathrm{O}_{3}$ & 14.71 & 29.49 & 2.31 & 15.91 & 16.91 & 16.6 \\
\hline $\mathrm{Fe}_{2} \mathrm{O}_{3}$ & 9.19 & 0.39 & 77.95 & 8.9 & 12.36 & 12.33 \\
\hline $\mathrm{MgO}$ & 14.85 & 0.08 & 2.99 & 2.53 & 5.69 & 5.64 \\
\hline $\mathrm{MnO}$ & 0.06 & - & - & 0.15 & 0.19 & 0.2 \\
\hline $\mathrm{CaO}$ & 12.89 & 16.28 & - & 4.21 & 7.69 & 7.99 \\
\hline $\mathrm{Na}_{2} \mathrm{O}$ & 0.95 & 3.57 & - & 5.08 & 2.96 & 4 \\
\hline $\mathrm{K}_{2} \mathrm{O}$ & 1.09 & 0.67 & - & 1.96 & 2.14 & 1.56 \\
\hline \multicolumn{7}{|c|}{ Residual sum of squares $(\Sigma R 2)=0.84$} \\
\hline \multicolumn{7}{|c|}{ Stage no. 2 The composition of andesites was assumed to be the parental liquid; the composition of decites was assumed to be the derivative } \\
\hline & & & & D2-Da & P2-An & $\mathrm{CP} 2$ \\
\hline $\mathrm{SiO}_{2}$ & 52.79 & 57.01 & 0.79 & 68.88 & 59.98 & 59.98 \\
\hline $\mathrm{TiO}_{2}$ & 0.96 & - & 5.36 & 0.58 & 1.27 & 1.08 \\
\hline $\mathrm{Al}_{2} \mathrm{O}_{3}$ & 2.61 & 27.19 & 0.29 & 16.04 & 15.91 & 15.83 \\
\hline $\mathrm{Fe}_{2} \mathrm{O}_{3}$ & 14.19 & 0.24 & 88.49 & 3.87 & 8.9 & 8.96 \\
\hline $\mathrm{MgO}$ & 14.93 & - & - & 0.69 & 2.53 & 2.39 \\
\hline $\mathrm{MnO}$ & 0.19 & - & - & 0.09 & 0.15 & 0.4 \\
\hline $\mathrm{CaO}$ & 11.99 & 9.06 & - & 1.49 & 4.21 & 4.32 \\
\hline $\mathrm{Na}_{2} \mathrm{O}$ & 0.07 & 5.61 & - & 6.26 & 5.08 & 5.29 \\
\hline $\mathrm{K}_{2} \mathrm{O}$ & 0.12 & 0.79 & - & 2.08 & 1.96 & 1.75 \\
\hline \multicolumn{7}{|c|}{ Residual sum of squares $(\Sigma R 2)=0.19$} \\
\hline
\end{tabular}

